REVISTA CIENCIAS BIOMÉ DICAS

PRESENTACIÓN DE CASOS CLÍNICOS

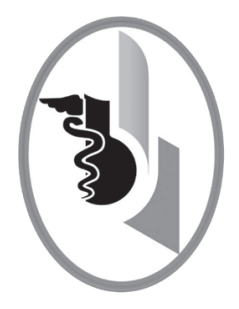

\title{
NEUROFIBROMA SOLITARIO GIGANTE DE LA CAVIDAD ORAL CON EXTENSIÓN AL CUELLO: REPORTE DE UN CASO
}

\author{
GIANT SOLITARY NEUROFIBROMA OF THE ORAL CAVITY WITH \\ EXTENSION TO THE NECK: CASE REPORT
}

\author{
Alvarado-Namén Nelly $A^{1}$ \\ Cáez-Ruiz Karina ${ }^{2}$ \\ Mendoza-Suárez Liney² \\ Redondo-Bermúdez César ${ }^{3}$ \\ Correspondencia: alejandra.alvaradonamen@gmail.com \\ Recibido para evaluación: marzo - 15 - 2013. Aceptado para publicación: octubre -25 - 2015.
}

\section{RESUMEN}

Introducción: el neurofibroma es un tumor benigno derivado de las fibras nerviosas muy infrecuente en la cavidad oral.

Caso clínico: paciente masculino con neurofibroma gigante originado en la cavidad oral con extensión al cuello. Fue manejado quirúrgicamente con resección completa, sin presentar recidiva tumoral. Se hizo diagóstico histopatológico y confirmación con inmunohistoquimica.

Conclusión: a pesar de ser infrecuentes en la cavidad oral, los neurofibromas deben sospecharse cuando el tumor tiene estrecha relación con el paquete vasculonervioso dentario inferior. Rev.cienc.biomed. 2015;6(2):376-380

\section{PALABRAS CLAVE}

Neurofibroma solitario; Cavidad oral; Mandíbula; Labiomandibulotomía.

\section{SUMMARY}

Introduction: neurofibroma is a benign tumor derived from nerve fibers is, very infrequent in the oral cavity.

Case report: male with a giant neurofibroma originated in the oral cavity that spread to the neck. The patient was managed with surgery, complete resection, without recurrence. It was diagnosed with histopathology test and was confirmed by immunohistochemical exam.

Conclusion: Although the neurofibromas are infrequent in the oral cavity, it has to be suspected when tumor has close relation with the inferior alveolar neurovascular bundle. Rev.cienc.biomed. 2015;6(2):376-380

\section{KEYWORDS}

Solitary neurofibroma; Mouth; Mandible; Labiomandibulotomy.

Médico. Estudiante de Postgrado. Otorrinolaringología. Facultad de Medicina. Universidad de Cartagena. Cartagena. Colombia.

Médico. Estudiante de Postgrado. Patología. Facultad de Medicina. Universidad de Cartagena. Cartagena. Colombia.

3 Médico. Especialista en Patología. Docente Departamento de Diagnóstico. Facultad de Medicina. Universidad de Cartagena. Cartagena. Colombia. 


\section{INTRODUCCIÓN}

El neurofibroma es un tumor benigno derivado de las fibras nerviosas compuesto por células de Schwann, células perineurales y fibroblastos. Aunque es de los tumores neurogénicos más comunes, es muy infrecuente en la cavidad oral. Puede presentarse como lesiones solitarias o múltiples. En un $90 \%$ de los casos se asocia a neurofibromatosis tipo I o enfermedad de Von Recklinghausen, por lo cual la presencia de un caso aislado requiere de un examen completo y de la indagación de la historia familiar con el fin de excluir la enfermedad. También, puede asociarse al síndrome poliglandular MEN III. De forma infrecuente se localizan en la cabeza y en el cuello, su sitio más común es la mandíbula por la presencia del paquete vasculonervioso dentario inferior. Se han reportado pocos casos de lesión intraósea, el tratamiento de elección es la escisión quirúrgica completa y las tasas de recidiva son muy bajas (1-5).

\section{REPORTE DE CASO}

Paciente masculino de 56 años, fumador pesado consultó el servicio de urgencias del Hospital Universitario del Caribe por presentar disnea y disfagia, secundario a una tumoración en cavidad oral de crecimiento lento, no dolorosa de más de 15 años de evolución, que apareció posterior a una exodoncia (Figura No 1).

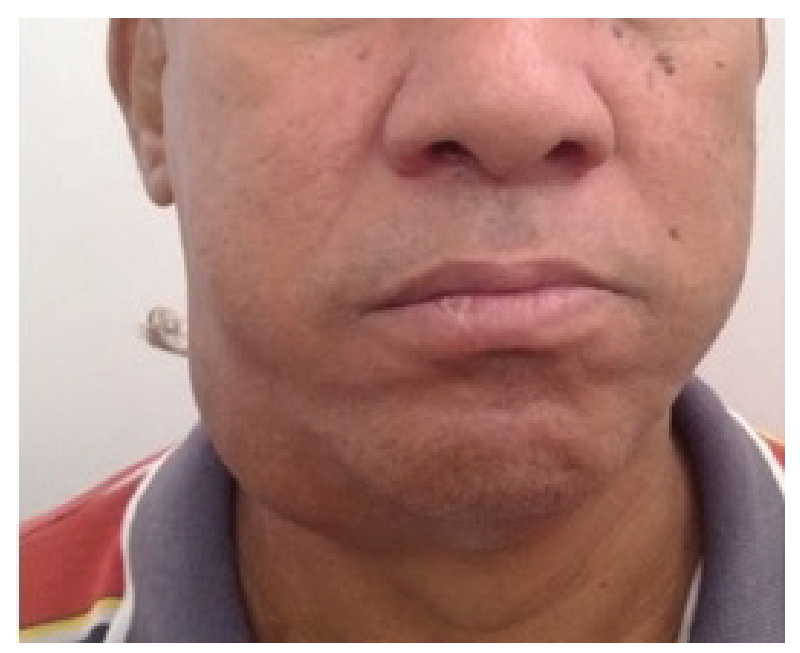

Figura No 1. Se aprecia al paciente con lesión en el cuello.
El paciente requirió traqueostomía y gastrostomía de urgencia. Posteriormente, fue valorado en otorrinolaringología donde se encontró que no presentaba compromiso de su estado general; en el examen se evidenció una masa submucosa de consistencia dura anclada en el trígono retromolar derecho que ocupaba la luz de la orofaringe y que respetaba el paladar blando. Desplazaba la lengua hacia la izquierda sin comprometer la movilidad de esta, ni la apertura oral

La lesión se extendía hacia el cuello donde se palpaba una masa dura, lisa en los niveles II y III. No se palparon adenopatías cervicales. La nasofibrolaringoscopia reportó la lesión descrita en orofaringe, por otro lado, la radiografía panorámica mostró radiolucidez a nivel del ángulo derecho de la mandíbula y calcificaciones en tejidos blandos (Figura No 2).

La tomografía contrastada del cuello evidenció una masa de aspecto heterogéneo que realzaba con el medio de contraste y ocupaba la orofaringe desde donde se proyectaba hacia la mandíbula, desplazando la glándula submaxilar y ocupando el espacio parafaríngeo produciendo efecto compresivo en la yugular interna (Figura No 3 ).

El estudio histopatológico de una biopsia incisional reportó una lesión benigna de linaje mesenquimal constituida por una proliferación de células fusiformes y uniformes dispuestas en fascículos, con atipia ligera, sin necrosis ni formas mitóticas, dispuestas en un estroma mixoide. La inmunohistoquímica mostró reactividad para S100 y CD34, un índice de proliferación medido con Ki67 del $5 \%$. No hubo reactividad para CD68. Estos hallazgos concluyeron que era un tumor neural benigno compatible con neurofibroma (Figura No 4).

Mediante abordaje externo combinado de labiomandibulotomía mandibular ("swing") y cervicotomía se resecó el tumor. Durante el procedimiento se evidenció que la lesión provenía del nervio alveolar inferior por lo que se realizó mandibulectomía marginal logrando resección completa de la lesión (Figura $N^{\circ} 5$ ). Posterior a la resección fue posible retirar la traqueostomía y la gastrostomía, 


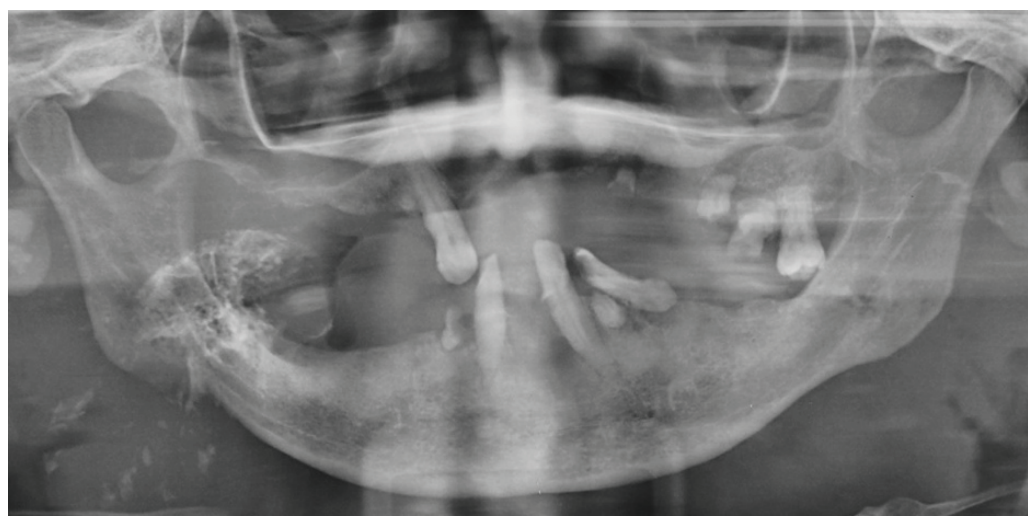

Figura No 2. Radiografía panorámica.

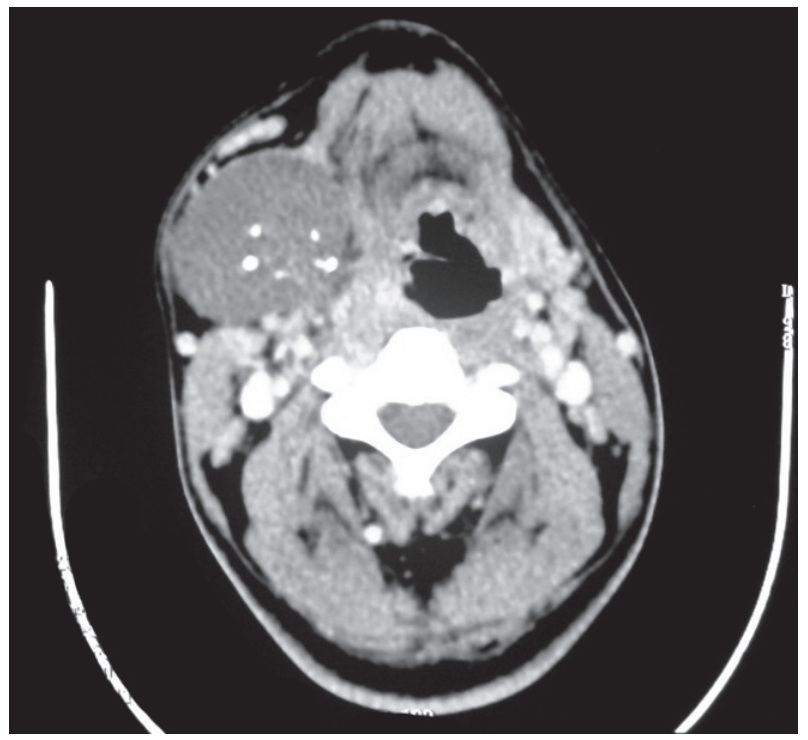

Figura No 3. Tomografía contrastada de cuello, corte axial.

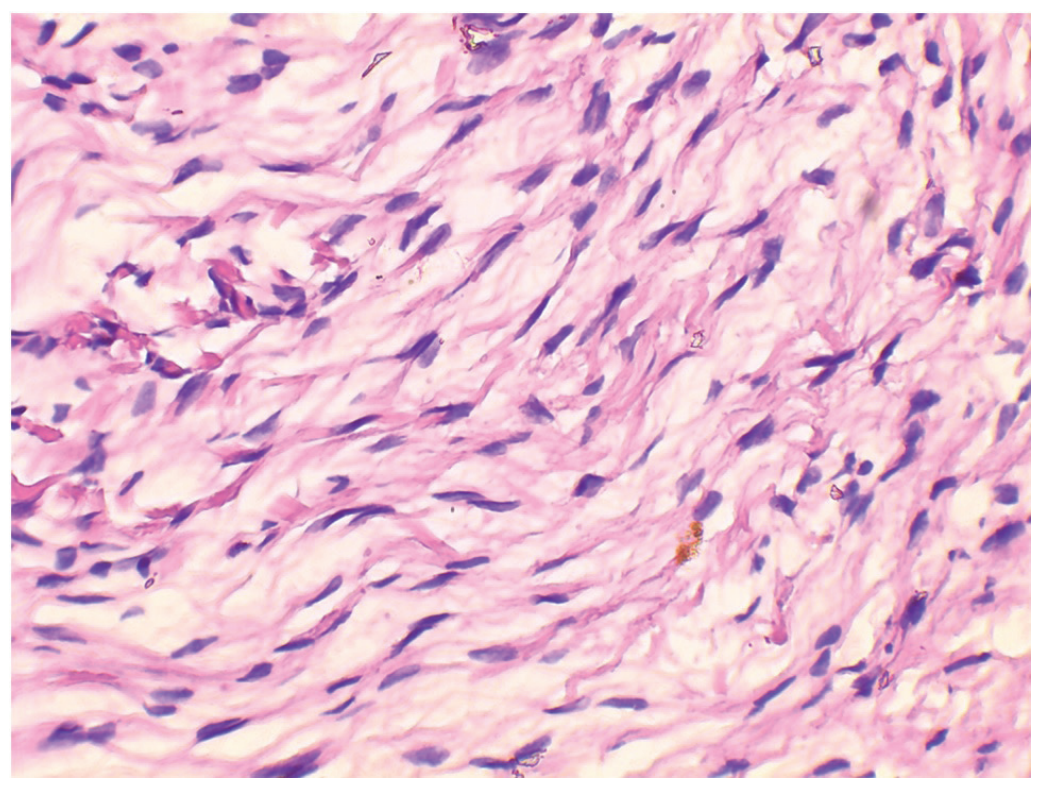

Figura No 4. Estudio histopatológico, coloración H-E. no se había observado recidiva tumoral ocho meses después de la intervención.

\section{DISCUSIÓN}

El neurofibroma es un tumor benigno derivado de las fibras nerviosas compuesto por células de Schwann, células perineurales y fibroblastos. Es muy infrecuente en la cabeza y cuello, más común es en la mandíbula por la presencia del paquete vasculonervioso dentario inferior. La edad media de presentación del neurofibroma es de 27.5 años con un rango entre 14 y 45 años, no tiene predilección por género. La primera descripción de un neurofibroma en la cavidad oral fue realizada por Bruce en 1954 y desde entonces, se han reportado pocos casos.

Los que surgen en la cavidad oral se caracterizan por ser lesiones pediculadas 0 nódulos sésiles, de crecimiento lento, indoloros, aunque cuando producen compresión nerviosa pueden ser dolorosos o causar parestesias. Su localización intraoral más frecuente es la lengua, seguido del paladar, los carrillos, el reborde alveolar edéntulo, las encías y el piso de la boca (1-5). Es llamativo en nuestro caso el tiempo de evolución y el tamaño, ya que se trataba de una lesión gigante originada en la cavidad oral con extensión al cuello, lo que sugirió desde un principio la naturaleza benigna del tumor.

Macroscópicamente los neurofibromas se caracterizan por ser lesiones blanquecinas de superficie lisa brillante. El diagnóstico definitivo se realizó con el estudio histopatológico, el cual mostró un patrón irregular 


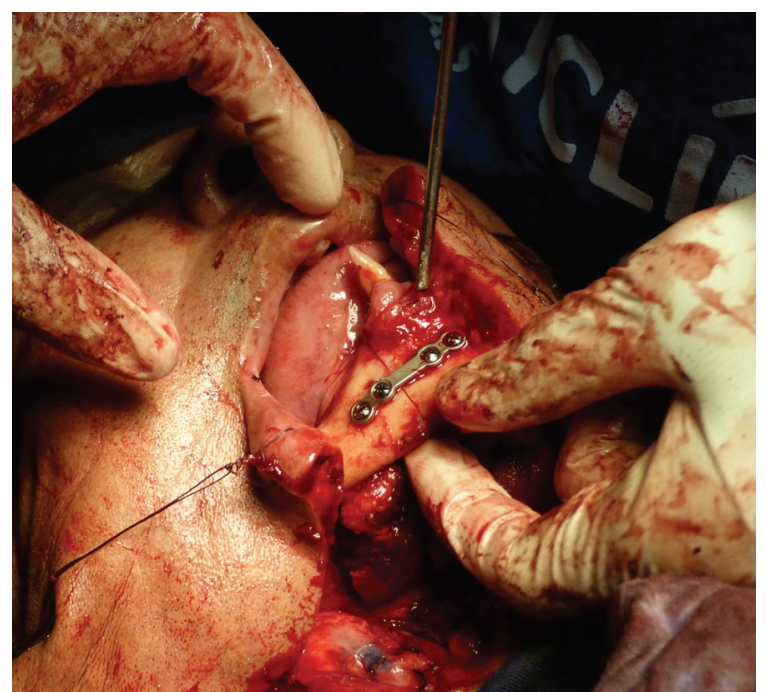

Figura 5. Labiomandibulotomia, material de osteosíntesis en posición para garantizar afrontamiento al culminar procedimiento

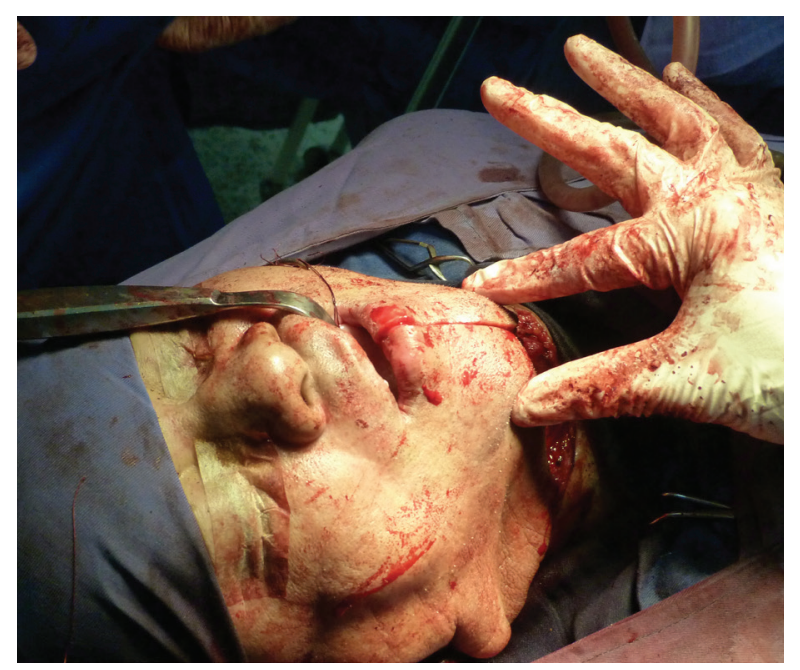

Figura 6. Nótese la importancia de garantizar el afrontamiento adecuado del borde del labio para obtener resultados estéticos.

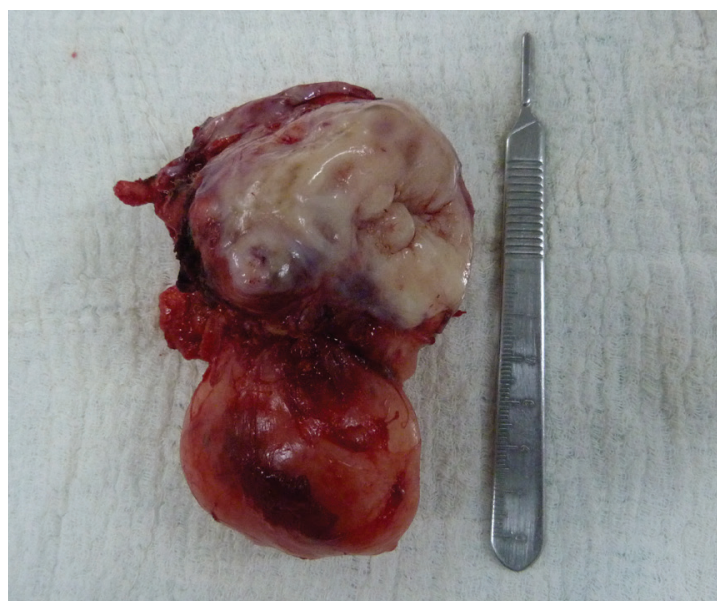

Figura 7. Aspecto macroscópico del tumor. de células fusiformes en un estroma compuesto por fibras colágenas y pequeños axones que se identificaron con tinción de plata. Los neurofibromas son inmunopositivos para proteína S-100 en 85 a 100\% de los casos, lo cual indica su origen neural $(4,5)$.

Una variante histológica muy rara con presencia de cuerpos de Meissner ha sido reportada (7). En este caso la positividad para S-100 apoyó el origen neurógeno del tumor.

El tratamiento de elección es la resección quirúrgica completa, con ella se intenta conservar el nervio del cual se origina el tumor. Dado que la escisión es completa se ha estudiado el tratamiento con terapia fotodinámica, por la gran morbilidad que acarrea, usando como agente fotosensibilizador el mtetrahidroxifenilclorado (mTHCP), con lo que se evidenció disminución significativa del tamaño del tumor, más que la desaparición del mismo (8). Así las cosas, los neurofibromas tienen excelente pronóstico, aunque han reportado recurrencias altas cuando son solitarios y se localizan en la cabeza o el cuello (6).

\section{CONCLUSIÓN}

A pesar que los neurofibromas son infrecuentes en la cavidad oral, siempre se debe tener en cuenta que los tumores neurogénicos presentan posibilidad diagnóstica cuando su localización es cercana al paquete vasculonervioso dentario inferior, y más aun, cuando comprometen la mandíbula. El diagnóstico definitivo de estos tumores se realiza únicamente con el estudio histopatológico y se confirma con la inmunohistoquímica. El tratamiento de elección es quirúrgico y la recidiva es muy baja cuando se logra una resección completa como podemos apreciar en nuestro caso.

CONFLICTOS DE INTERESES: ninguno que declarar.

FINANCIACIÓN: recursos propios de los autores. Costos hospitalarios, estudios de laboratorio, valoraciones por imágenes, insumos clínicos y honorarios profesionales fueron cubiertos dentro del proceso asistencial. 


\section{REFERENCIAS BIBLIOGRÁFICAS}

1. Vivek N, Manikandhan R, James PC, Rajeev R. Solitary intraosseous neurofibroma of mandible. Indian J Dent Res Off Publ Indian Soc Dent Res. 2006;17(3):135-8.

2. Deichler J, Martínez R, Niklander S, Seguel H, Marshall M, Esguep A. Solitary intraosseous neurofibroma of the mandible. A propos of a case. Med Oral Patol Oral Cirugía Bucal. $2011 ; 16(6): 704-7$.

3. Tao Q, Wang Y, Zheng C. Neurofibroma in the left mandible: A Case Report. Kaohsiung J Med Sci. $2010 ; 26(4): 217-21$.

4. Depprich R, Singh DD, Reinecke $P$, Kübler NR, Handschel J. Solitary submucous neurofibroma of the mandible: review of the literature and report of a rare case. Head Face Med. 2009; 5:24.

5. Dalili Z, Adham G. Intraosseous Neurofibroma and concurrent involvement of the mandible, maxilla and orbit: report of a case. Iran J Radiol. $2012 ; 9(1): 45-9$.

6. Polak M, Polak G, Brocheriou C, Vigneul J: Solitary neurofibroma of the mandible: case report and review of the literature. J Oral Maxillofac Surg 1989, 47:65-68.

7. Ohno J., Iwahashi T., Ozasa R., Okamura K., Kunihisa T. Solitary neurofibroma of the gingiva with prominent differentiation of Meissner bodies: a case report. Diagnostic Pathology $2010,5: 61$.

8. Hamdoon Z., Jerjes W., Al-Delayme R., Hopper C. Solitary giant neurofibroma of the neck subjected to photodynamic therapy: case study. Head \& Neck Oncology 2012, 4: 30. de Cartagena Fundada en 1827

REVISTA CIENCIAS BIOMÉDICAS
Es el órgano de información científica de la Facultad de Medicina de la Universidad de Cartagena. Colombia.

Publique su trabajo en esta revista enviando su manuscrito a: revistacienciasbiomedicas@unicartagena.edu.co www. revistacienciasbiomedicas.com www.revistacienciasbiomedicas.com.co

Revista ciencias Biomédicas es una publicación independiente, imparcial, abierta, revisada por pares, de elevada visibilidad internacional, con circulación online e impresa. Publica artículos en todas las modalidades universalmente aceptadas en inglés y en español, de temas referentes con todas las ciencias biomédicas, incluyendo ámbitos clínicos, epidemiológicos o de estudios básicos.

El sistema de gestión de manuscritos es rápido y justo.

Revista Ciencias Biomédicas está incluida en varias bases de datos latinoamericanas e internacionales.

Antes de enviar su manuscrito, revise las recomendaciones para los autores, presentes en

www. revistacienciasbiomedicas.com

www.revistacienciasbiomedicas.com.co 